\title{
Long-term effects of a transdiagnostic protocol in reducing symptoms of anxiety and depression in young Spanish-speaking children
}

\section{Fernández-Martínez, I., Morales, A., Espada, J. P., Gonzálvez, M. T., \& Orgilés, M.}

Miguel Hernández University, Spain

\section{BACKGROUND}

\section{- Effective programs targeting anxiety and depressive symptoms in Spanish children since early years remain scarce. Super Skills for Life (SSL) is a recent transdiagnostic indicated prevention protocol designed to reduce these comorbid internalizing symptoms in only eight weeks. SSL is an innovative protocol consisting of eight 45- minute sessions, once a week, and promotes the development of the following skills: education in emotions and feelings, relaxation techniques, cognitive reappraisal, social competence, problem- solving, and behavior activation. SSL can be delivered in the school setting and to small groups of children.}

The aim of this research was to examine the effects of SSL in reducing anxiety and depressive symptoms in young Spanishspeaking children in the 12-month follow-up.

\section{METHODS}

Participants were 123 children aged 6 to 8 years $(M$ $=6.89 ; S D=0.79 ; 46.7 \%$ girls $)$ and their parents $(21.1 \%$ males $)$

Recruited from 10 schools located in the southeast of Spain (randomly assigned to a control group (CG; $n=5)$ and intervention group (IG; $n=5)$ )

At the baseline and 12-month post-intervention (87\% retention) parents completed the following measures:

Spence Children's Anxiety Scale - Parent version (SCAS-P; Nauta et al., 2004)

Mood and Feelings Questionnaire - Parent version (MFQ-P; Angold et al., 1995)

Generalized estimation equations and adjusted odds ratio (AOR) were estimated. SPSS v25.

\section{RESULTS}

$\square$ At twelve months after the intervention, SSL had a statistically significant effect on overall depression and anxiety symptoms, but also on other specific anxiety symptoms as measured by SCAS-P (Table 1).

$\square$ Figure 1 shows changes between baseline and one-year post-intervention scores for general measures of anxiety and depression.

r
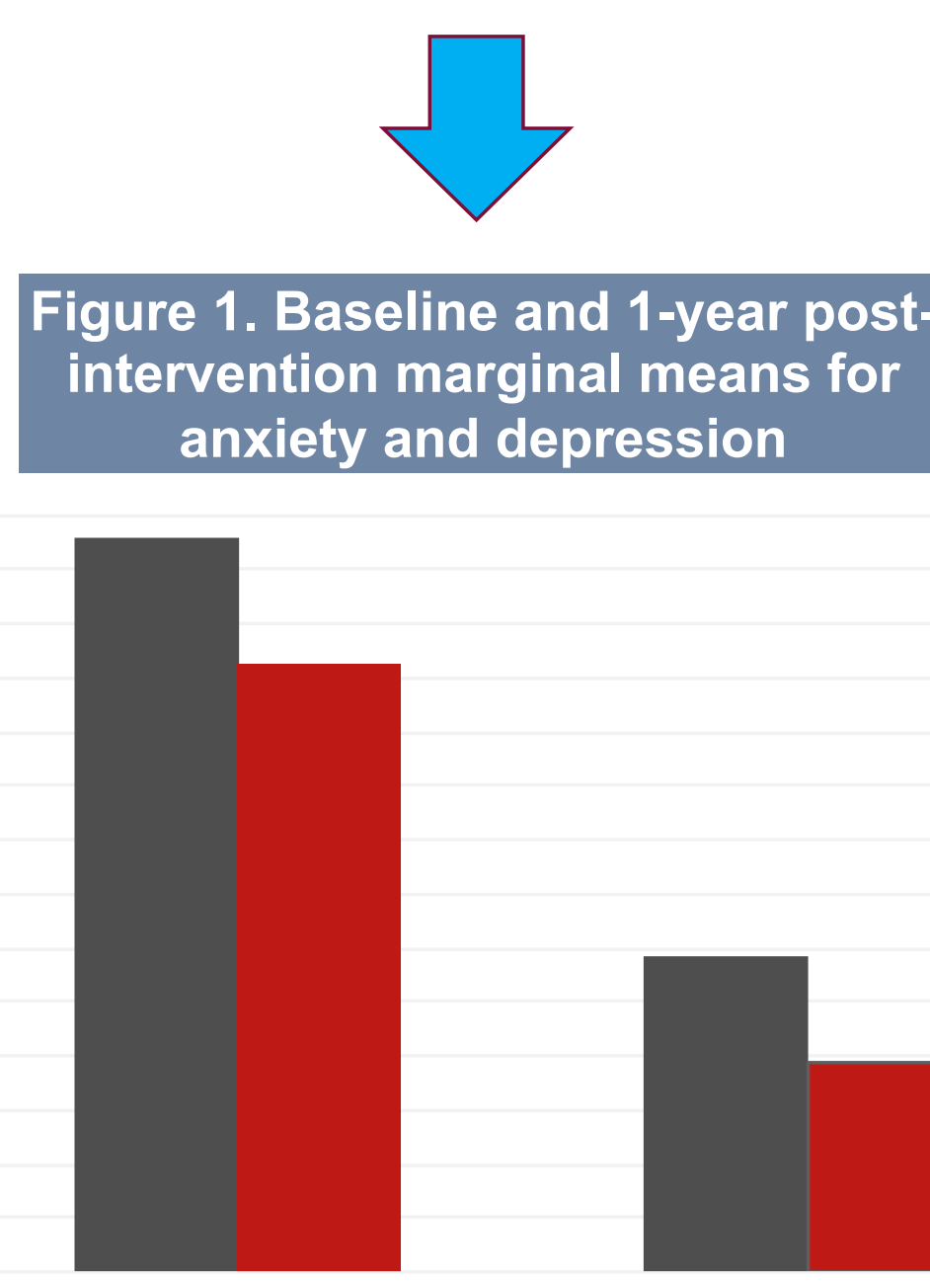

Anxiety

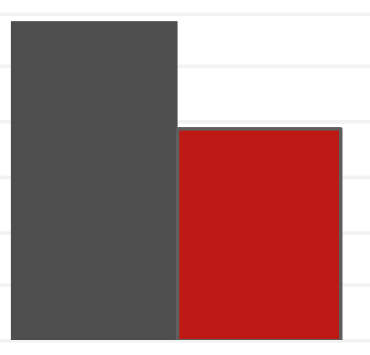

Depression

- Baseline a12-month post-intervention

\begin{tabular}{|c|c|c|}
\hline \multicolumn{3}{|l|}{ Outcomes } \\
\hline & AOR $(95 \% \mathrm{Cl})$ & $P$ value \\
\hline \multicolumn{3}{|l|}{ MFQ-P } \\
\hline Overall Depression & $.01(.001, .22)$ & .004 \\
\hline \multicolumn{3}{|l|}{ SCAS-P } \\
\hline Overall Anxiety & $.002(.001, .18)$ & .008 \\
\hline Social Anxiety & $.22(.06, .76)$ & .01 \\
\hline Physical injury fears (specific phobia) & $.26(.10, .70)$ & .007 \\
\hline Panic/Agoraphobia & $.21(.07, .63)$ & .005 \\
\hline
\end{tabular}

This research provides initial support for the long-lasting effectiveness of SSL for reducing anxiety and depressive symptoms in young Spanish children. SSL may be a promising tool for reducing emotional symptoms at early years and prevent the onset of future disorders.

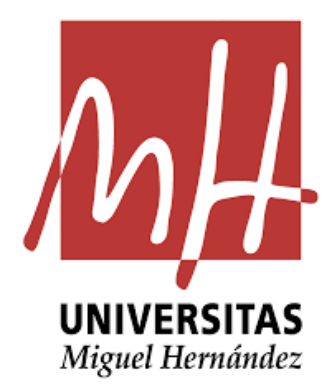

More info

\section{aitana}

